\title{
Inhibition of Contractile Activity During Postconditioning Enhances Cardioprotection by Restoring Sarcolemmal Dystrophin Through Phosphatidylinositol 3-Kinase
}

\author{
Akira Moriguchi, MD; Hajime Otani, MD; Kei Yoshioka, MD; Takayuki Shimazu, MD; \\ Masanori Fujita, MD; Toru Okazaki, MD; Daisuke Sato, MD; \\ Shiori Kyoi, MD; Toshiji Iwasaka, MD
}

\begin{abstract}
Background: Although ischemic postconditioning (IPost) confers cardioprotection by protecting the mitochondria though the activation of phosphatidylinositol 3-kinase (PI3K), a potential drawback of IPost is impairment of aerobic ATP generation during reperfusion by repeated ischemia. This decrease in ATP might inhibit the restoration of sarcolemmal dystrophin, which is translocated during ischemia, and render cardiomyocytes susceptible to contraction-induced oncosis.
\end{abstract}

\begin{abstract}
Methods and Results: Isolated rat hearts were subjected to $30 \mathrm{~min}$ ischemia and $120 \mathrm{~min}$ reperfusion. IPost induced by 20 cycles of 10-s reperfusion and 10-s ischemia enhanced the activation of PI3K as evidenced by the increased phosphorylation of Akt, but had no effect on myocardial ATP, restoration of sarcolemmal dystrophin, or cardiomyocyte oncosis during IPost. Administration of the contractile blocker, 2,3-butanedione monoxim (BDM), during IPost increased myocardial ATP and facilitated the redistribution of dystrophin to the sarcolemma. This led to reduced cardiomyocyte oncosis and infarct size, and improved the left ventricular function. The anti-oncotic effect of BDM occurred without changing the anti-apoptotic effect of IPost. The PI3K inhibitor, LY294002, prevented the phosphorylation of Akt, decreased the recovery of ATP and restoration of sarcolemmal dystrophin, and blocked the anti-oncotic and anti-apoptotic effects of IPost.
\end{abstract}

Conclusions: These results suggest that the inhibition of contractile activity during IPost prevents cardiomyocyte oncosis and enhances cardioprotection through PI3K-dependent restoration of sarcolemmal dystrophin. (Circ J 2010; 74: 2393-2402)

Key Words: Dystrophin; Phosphatidylinositol 3-kinase; Postconditioning

$\mathbf{I}$ schemic postconditioning (IPost) is a series of brief mechanical interruptions of reperfusion following a specific prescribed algorithm applied at the very onset of reperfusion. ${ }^{1}$ IPost is a potentially promising approach to limit infarct size during the revascularization of patients with acute myocardial infarction (MI)., ${ }^{2,3}$ The cardioprotective mechanism of IPost involves the reduction of oxygen-derived free radicals, ${ }^{4,5}$ prolonged acidosis during early reperfusion ${ }^{6}$ and signal transductions ${ }^{1}$ that attenuates the multiple manifestations of reperfusion injury by inhibiting the opening of the mitochondrial permeability transition pore (mPTP), a nonspecific channel localized in the mitochondrial inner membrane. ${ }^{1,7}$ Opening of the mPTP promotes activation of death signaling involved in apoptosis and collapses the mitochondrial membrane potential, leading to a loss of ATP generation and necrosis. ${ }^{8-10}$ Among the distal signal transduction path- ways involved in the IPost-induced inhibition of mPTP opening and cardioprotection, the activation of reperfusion injury salvage kinases (RISK), such as phosphatidylinositol 3-kinase (PI3K)-Akt and ERK1/2, has been implicated in the mechanistic link between IPost and cardioprotection. ${ }^{11,12}$

\section{Editorial p 2295}

Although IPost confers a variety of protective effects on the mitochondria, it interrupts aerobic ATP generation during repeated ischemia, contributing to impairment of cellular repair processes following prolonged myocardial ischemia. One of the important roles of ATP generation at the time of reperfusion is to inhibit the oncosis of cardiomyocytes by preventing the rupture of their fragile sarcolemmal membranes. Oncosis is defined by cell death due to primary dis-

Received February 12, 2010; revised manuscript received June 17, 2010; accepted June 28, 2010; released online September 18, 2010

Time for primary review: 18 days

The Second Department of Internal Medicine, Kansai Medical University, Moriguchi, Japan

Mailing address: Hajime Otani, MD, The Second Department of Internal Medicine, Kansai Medical University, 10-15 Fimizono-cho,

Moriguchi 570-8507, Japan. E-mail: otanih@takii.kmu.ac.jp

ISSN-1346-9843 doi:10.1253/circj.CJ-10-0105

All rights are reserved to the Japanese Circulation Society. For permissions, please e-mail: cj@j-circ.or.jp 


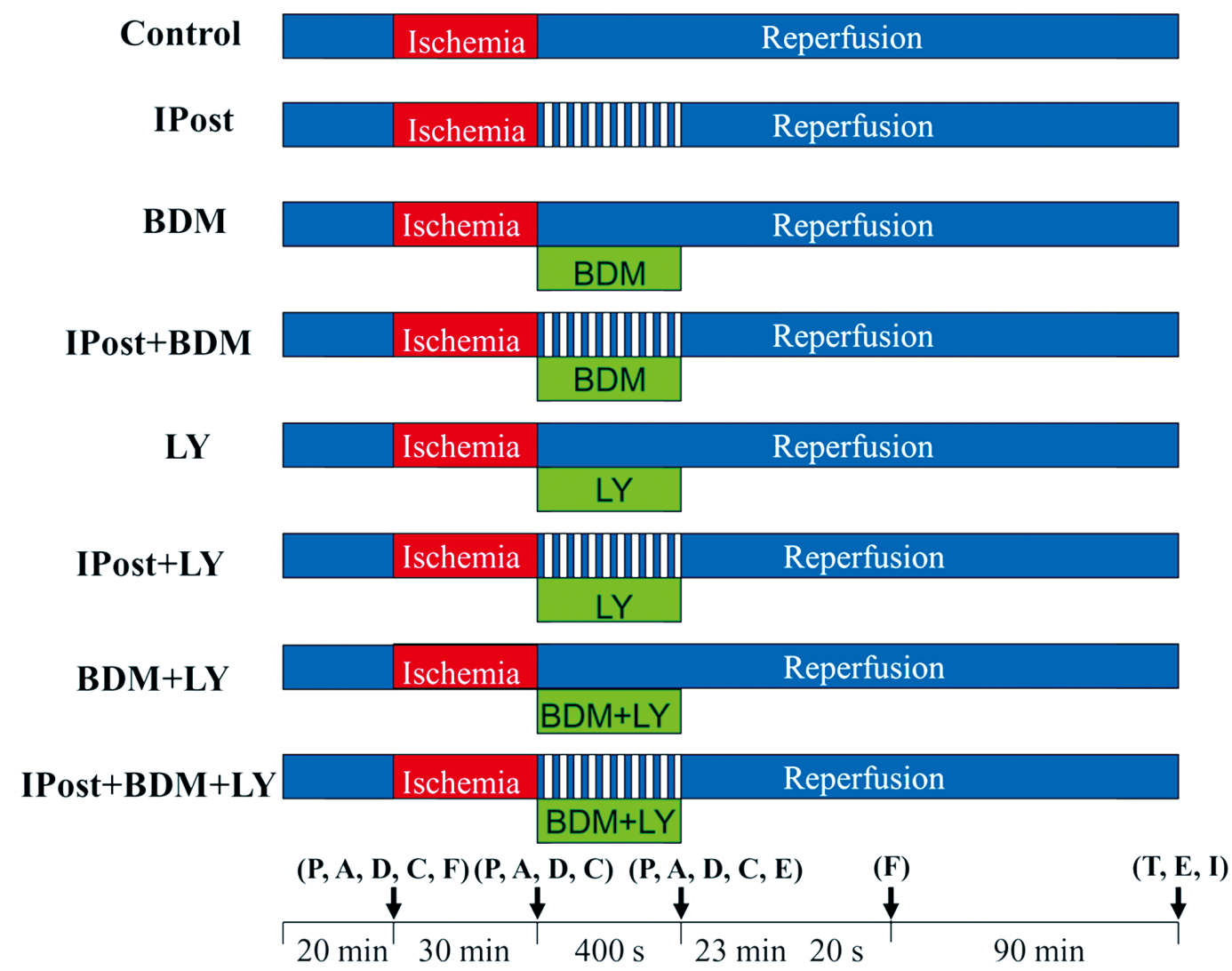

Figure 1. Experimental protocol. Isolated rat hearts were perfused on the Langendorff apparatus at a constant mean pressure of $70-75 \mathrm{mmHg}$ using a Krebs-Henseleit bicarbonate buffer solution for $20 \mathrm{~min}$. The control heart was then subjected to $30 \mathrm{~min}$ of global ischemia followed by 120 min of reperfusion. Ischemic postconditioning (IPost) consisted of 20 cycles of 10 -s reperfusion and 10-s ischemia. The contraction blocker, 2,3-butanedione monoxime (BDM), was administered upon reperfusion for a duration of 400 s, or was added during IPost. LY294002 (LY) was administered during control reperfusion or reperfusion with BDM for a duration of 400 s or during IPost in the presence or absence of BDM. Arrows indicate where measurements were carried out. P, phospho-Akt; A, ATP; D, dystrophin; C, caspase-3; F, left ventricular function; E, Evans blue; T, TUNEL; I, infarct size.

ruption of the plasma membrane..$^{13}$ Cardiomyocyte oncosis is induced by the reintroduction of contractile activity to the weakened cells at the time of reperfusion. ${ }^{14,15}$ Although degradation of a variety of cytoskeletal membrane proteins that are involved in the sarcolemmal integrity has been implicated in the membrane fragility, ${ }^{16}$ loss of sarcolemmal dystrophin has been singled out as a key mediator of this type of pathogenesis. ${ }^{17,18}$

Dystrophin provides a mechanically strong physical linkage between the sarcolemmal membrane and the costameric cytoskeleton in the cardiac muscle,${ }^{19}$ thereby stabilizing the sarcolemmal membrane against the shear stresses imposed during eccentric muscle contraction..$^{20,21} \mathrm{We}$ have previously reported that dystrophin is translocated from the sarcolemma during ischemia, ${ }^{22,23}$ and that redistribution of dystrophin to the sarcolemma during reperfusion depends on mitochondrial ATP generation. ${ }^{24}$ Therefore, despite the fact that IPost protects the mitochondria, it is anticipated that the inhibition of aerobic ATP generation during IPost is detrimental for the recovery of sarcolemmal integrity and renders cardiomyocytes susceptible to oncosis. Therefore, developing strategies that can protect cardiomyocytes depleted of dystrophin from oncosis during IPost might be necessary to enhance the efficacy of IPost.
The present study was designed to investigate whether inhibition of contractile activity during IPost can prevent cardiomyocyte oncosis and potentiate the infarct size-limiting effect of IPost. Our results suggest that inhibition of contractile activity during IPost increases myocardial ATP, facilitates restoration of sarcolemmal dystrophin, inhibits oncosis, and reduces the infarct size without changing protection against apoptosis.

\section{Methods}

\section{Animal Preparation and Perfusion Technique}

Male Sprague-Dawley rats weighing 250-300 g were used in the present study. All animals were handled in accordance with the "Guide for the Care and Use of Laboratory Animals" prepared by the Institute of the Care and Use of Laboratory Animal Resources, National Research Council, and published by the National Academy Press (revised 1996). The study was approved by the Animal Care Committee of Kansai Medical University (Moriguchi, Japan).

The experimental protocol is shown in Figure 1. The isolated and buffer-perfused rat heart preparation was established as described previously, ${ }^{23}$ and the heart was subjected to $30 \mathrm{~min}$ ischemia followed by $120 \mathrm{~min}$ reperfusion. IPost 
consisted of 20 cycles of 10-s reperfusion and 10-s ischemia. When the buffer contained the contractile blocker, 2,3butanedione monoxime (BDM; Sigma Chemical Co, Tokyo, Japan), at a concentration of $20 \mathrm{mmol} / \mathrm{L}$, we made an equimolar reduction in the concentration of $\mathrm{NaCl}$ in the buffer. This concentration of BDM has been shown to abolish contractility without an apparent toxic effect in the same experimental model. ${ }^{24}$ The PI3K inhibitor, LY294002 (Calbiochem, San Diego, CA, USA), was added at a concentration of $10 \mu \mathrm{mo} / \mathrm{L}$ into the buffers in the presence or absence of BDM for the first $400 \mathrm{~s}$ of reperfusion (equivalent to the duration of IPost).

In the experiments where cardiomyocyte oncosis was detected, Evans blue (EB) dye (Sigma) was added to the buffer at a concentration of $0.1 \%$ for $400 \mathrm{~s}$ at the time of reperfusion or at the last $5 \mathrm{~min}$ of reperfusion. EB was washed away with the same buffer without EB for $5 \mathrm{~min}$.

\section{Measurements of Left Ventricular (LV) Function}

Isovolumic LV function was measured as described previously. ${ }^{23}$ During the stabilization period, a latex balloon was inserted into the left ventricle through the left atrium to measure the isovolumic LV function. The balloon was filled with saline solution to produce an LV end-diastolic pressure (LVEDP) of $5-10 \mathrm{mmHg}$ at the baseline, and the balloon volume was kept constant throughout the experiment. Hearts with LV developed pressures (LVDP) less than $80 \mathrm{mmHg}$ or a heart rate less than 240 beats/min at the baseline were excluded from the study.

\section{Assay for PI3K Activity}

The PI3K activity in the heart was evaluated by a Western blot analysis of phospho-Akt. ${ }^{25}$ Frozen heart samples were ground with lysis buffer containing $20 \mathrm{mmol} / \mathrm{L}$ Tris $(\mathrm{pH} 7.5)$, $150 \mathrm{mmol} / \mathrm{L} \mathrm{NaCl}, 1 \mathrm{mmol} / \mathrm{L}$ EDTA, $1 \mathrm{mmol} / \mathrm{L}$ EGTA, $1 \%$ Triton $\mathrm{X}-100,2.5 \mathrm{mmol} / \mathrm{L}$ sodium pyrophosphate, $1 \mathrm{mmol} / \mathrm{L}$ $\beta$-glycerophosphate, $1 \mathrm{mmol} / \mathrm{L} \mathrm{Na}_{3} \mathrm{VO}_{4}, 1 \mathrm{mmol} / \mathrm{L} \mathrm{PMSF}$, and a protease inhibitor cocktail (Complete, Roche Molecular Biochemicals, Mannheim, Germany). The homogenates were centrifuged at $37^{\circ} \mathrm{C}$ for $5 \mathrm{~min}$ at $14,000 \mathrm{~g}$, and equal amounts of supernatants $(50 \mu \mathrm{g})$ were resolved on $7.5 \%$ SDS-polyacrylamide gels. The protein concentration was determined with a Bio-Rad protein assay kit (Bio-Rad Laboratories, Hercules, CA, USA). Electroblotting procedures were used to transfer proteins to nitrocellulose membranes (Bio-Rad). Nitrocellulose membranes were blocked with a buffer containing $50 \mathrm{mmol} / \mathrm{L}$ Tris, $\mathrm{pH} 7.6,150 \mathrm{mmol} / \mathrm{L} \mathrm{NaCl}, 0.1 \%$ Tween 20 , and $5 \%$ non-fat dry milk for $1 \mathrm{~h}$ at room temperature and incubated overnight at $4^{\circ} \mathrm{C}$ with the antibodies against rabbit polyclonal Akt or rabbit polyclonal phosphorylated Akt (Ser473) obtained from Cell Signaling (Beverly, MA, USA). Immunolabeling was detected by enhanced chemiluminescence (Amersham Pharmacia Biotech, Piscataway, NJ, USA) according to the manufacturer's instructions, and was quantified by densitometric analysis using the image analyzing software system, Win Roof (Mitani, Fukui, Japan).

\section{Measurement of Myocardial ATP Content}

The hearts were quickly frozen in liquid nitrogen, and the ATP content of the LV muscle was measured, as described previously. ${ }^{24}$

\section{Immunofluorescence Microscopy for Dystrophin}

Myocardial tissue slices of 2-mm thickness were obtained from the mid-LV level at the indicated time-points, frozen sections were made, and immunofluorescence microscopy for dystrophin was performed, as described previously. ${ }^{23}$ Briefly, frozen samples were cut into $\sim 6 \mu \mathrm{m}$ sections and were mounted on glass slides, incubated in acetone and hydrogen peroxide, rinsed with PBS, and blocked with $10 \%$ normal rabbit serum. The sections were incubated for $1 \mathrm{~h}$ at room temperature with mouse monoclonal anti-dystrophin antibodies (MANDRA-1; Sigma) at a dilution of 1:100 and washed with PBS. They were then incubated for $2 \mathrm{~h}$ at room temperature with fluorescein isothiocyanate (FITC)-conjugated rabbit anti-mouse immunoglobulin at a dilution of 1:100. To visualize the sarcolemma, sections stained for dystrophin were subjected to secondary staining with tetrarhodamine isothiocyanate (TRITC)conjugated wheat germ agglutinin (WGA; Sigma). The slides were viewed with a confocal laser microscope (Fluo View, Olympus, Tokyo, Japan). To quantify sarcolemmal dystrophin, 100 cardiomyocytes were randomly selected by an observer blind to the identity of the samples. The area of sarcolemma and the fluorescence intensity of dystrophin were then quantified using an image analyzing software program, as described previously. ${ }^{24}$

\section{Detection of Oncotic Cardiomyocytes}

Oncotic cardiomyocytes were detected by immunofluorescence microscopy for dystrophin and EB dye, as described previously. ${ }^{24}$ The number of EB-positive and -negative cardiomyocytes was counted for 60 high-power fields (magnification $\times 600$ ) from the endocardium through the epicardium of the mid-LV free wall, and the percentage of EB-positive cardiomyocytes was calculated.

\section{Caspase-3 Activity Assay}

At the indicated time-points, the experiment was terminated, and a ventricular muscle sample was taken and snap-frozen in liquid nitrogen. Caspase-3 activity was measured using a kit from MBL (Nagoya, Japan). The cardiac muscle lysate was prepared by homogenization of the frozen tissue sample using an ULTRA-TURLAX homogenizer in the lysis buffer provided with the kit. The lysates were centrifuged at $10,000 \mathrm{~g}$ for $10 \mathrm{~min}$ at $4^{\circ} \mathrm{C}$, and the supernatants containing $100 \mu \mathrm{g}$ protein were used for the caspase- 3 activity assay, as described previously. ${ }^{26}$

\section{Detection of Apoptotic Cardiomyocytes}

Cardiomyocyte apoptosis was evaluated using the TUNEL assay at the end of reperfusion, as described previously..$^{27}$ The frozen sections were prepared as described before and were stained with FITC fluorescence for TUNEL using an assay kit (Intergen, Purchase, NY, USA), as described by the manufacturer. Double-immunofluorescence staining was then performed using a mouse monoclonal anti- $\alpha$-actinin antibody (Sigma) and TRITC-labeled anti-mouse immunoglobulins (Sigma). The sections were visualized using a confocal laser microscope (Fluoview, Olympus), and the number of TUNELpositive cardiomyocytes was counted, as described previously. ${ }^{27}$

\section{Infarct Size Measurements}

Infarct size measurements were performed by using a triphenyltetrazolium chloride (TTC) (Sigma) staining method, as described previously. ${ }^{23}$

\section{Statistical Analysis}

All numerical data are expressed as the mean \pm SE. Statistical analysis was performed by using a 1-way or 2-way 

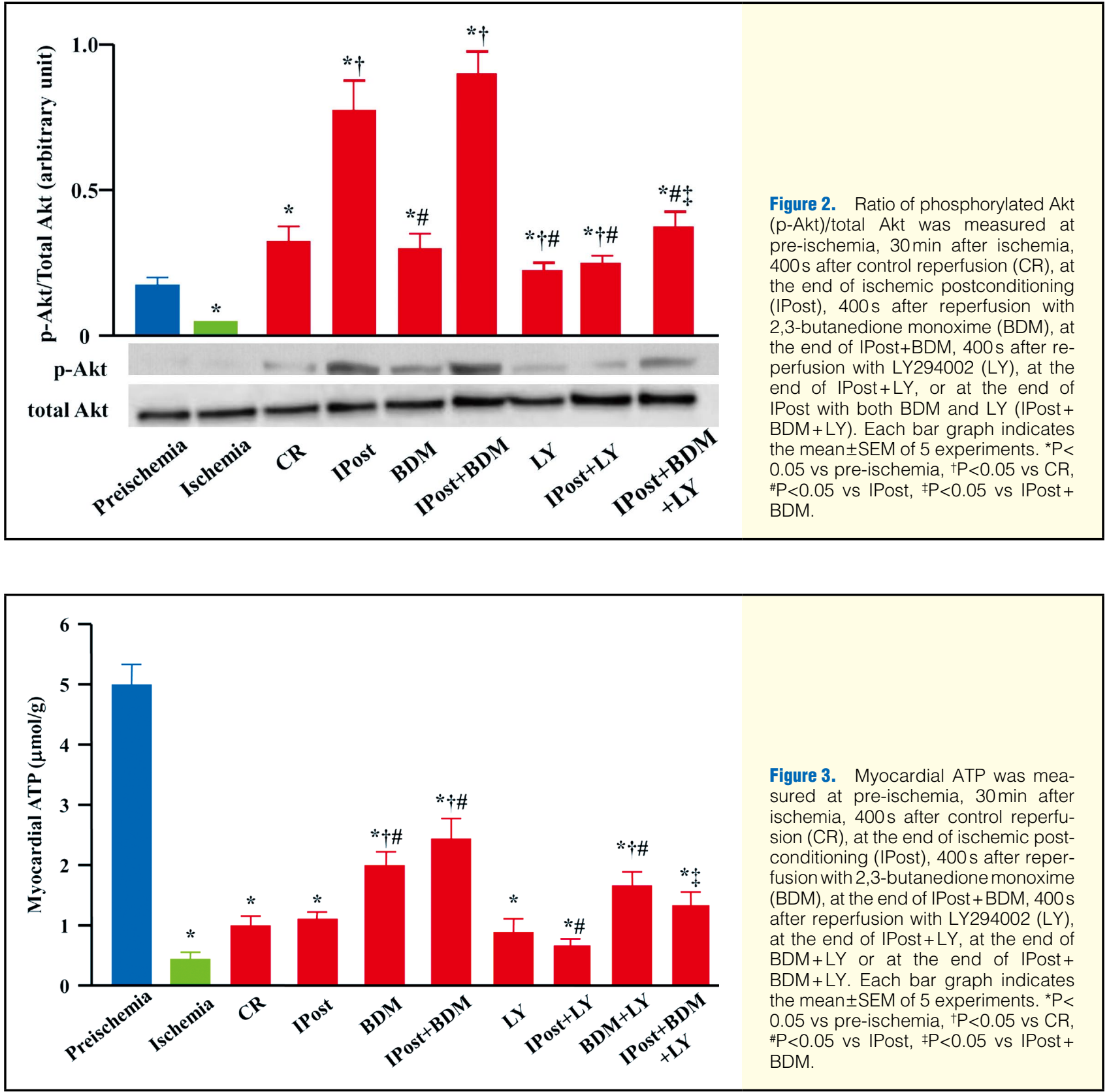

repeated measures ANOVA followed by the Fisher's PLSD post-hoc test. The differences were considered significant at a $P$ value of $<0.05$.

\section{Results}

\section{Effect of IPost and BDM on the Phosphorylation of Akt} During IPost

PI3K has been reported as an essential signaling element for IPost-mediated cardioprotection in the isolated and perfused rat heart. ${ }^{28,29}$ Therefore, we evaluated PI3K activity by measuring the phosphorylation of Akt/total Akt (Figure 2). The total Akt level did not change during ischemia and reperfusion under any of the treatment modalities. Phospho-Akt was significantly increased during control reperfusion compared to the pre-ischemic baseline, and was further increased by IPost $(\mathrm{P}<0.05$ compared to control reperfusion). The addition of BDM during control reperfusion or IPost had no significant effect on the level of phospho-Akt. As expected, the PI3K inhibitor, LY294002, abolished the increase in phospho-Akt that was observed during control reperfusion, IPost and IPost combined with BDM. LY294002 also inhibited the increase in phospho-Akt level during reperfusion with BDM (data not shown).

\section{Effect of IPost and BDM on Myocardial ATP}

Myocardial ATP was decreased during ischemia and remained at lower levels during reperfusion (Figure 3). Treatment with BDM during control reperfusion or IPost significantly increased myocardial ATP compared to control reperfusion or IPost alone. Although LY294002 had no significant effect on myocardial ATP during control reperfusion and reperfusion with BDM, it significantly decreased myocardial ATP during IPost and IPost combined with BDM. 


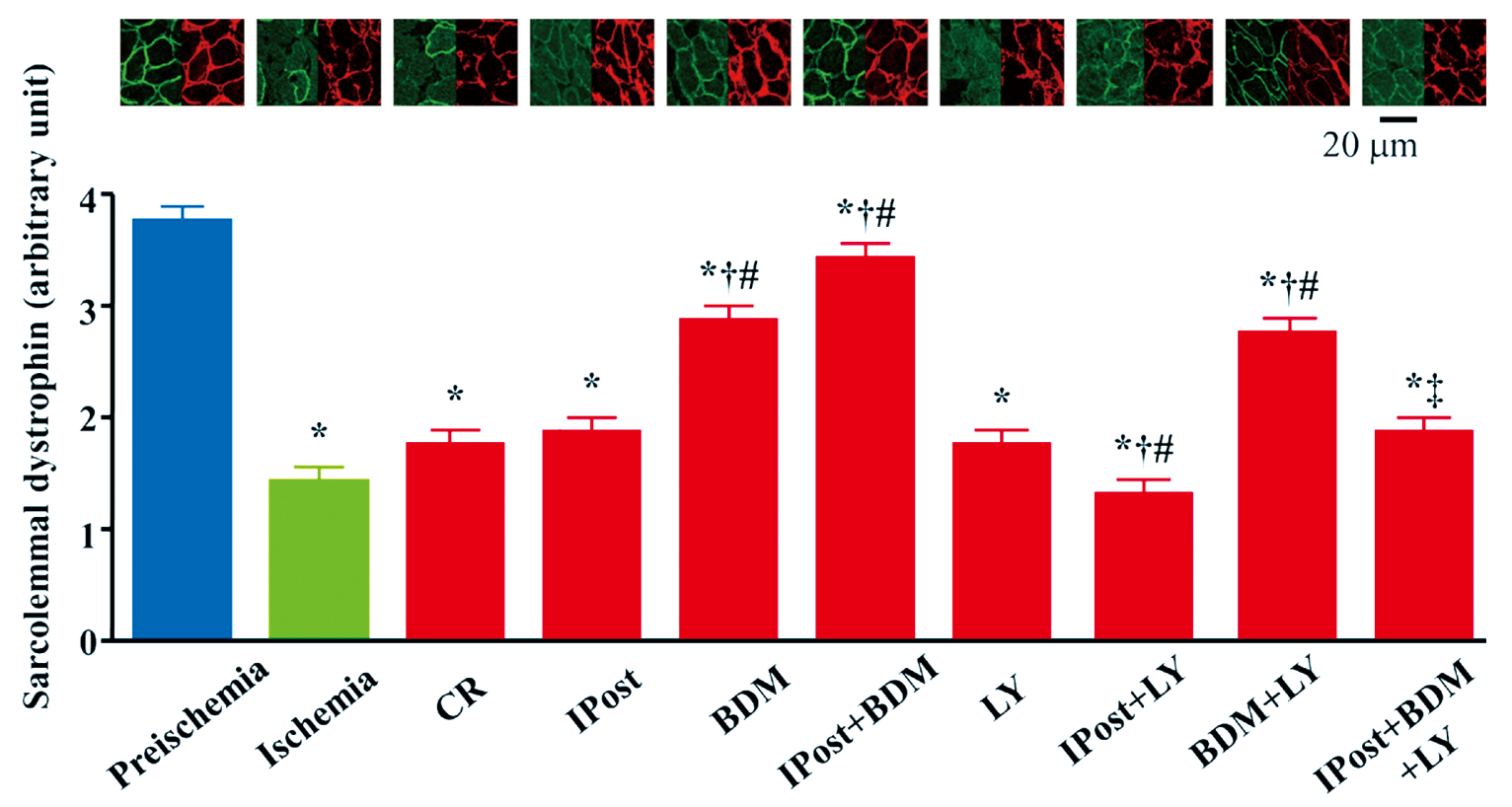

Figure 4. Immunofluorescence images of dystrophin (green) and wheat germ agglutinin (red). Sarcolemmal dystrophin was quantified at pre-ischemia, $30 \mathrm{~min}$ after ischemia, $400 \mathrm{~s}$ after control reperfusion (CR), at the end of ischemic postconditioning (IPost), 400 s after reperfusion with 2,3-butanedione monoxime (BDM), at the end of IPost+BDM, 400 s after reperfusion with LY294002 (LY), at the end of IPost $+L Y$, at the end of BDM + LY or at the end of IPost + BDM + LY. Each bar graph indicates the mean \pm SEM of 5 experiments. ${ }^{*} \mathrm{P}<0.05$ vs pre-ischemia, ${ }^{\dagger} \mathrm{P}<0.05$ vs $\mathrm{CR}$, ${ }^{*} \mathrm{P}<0.05$ vs IPost, ${ }^{\ddagger} \mathrm{P}<0.05$ vs IPost +BDM.

\section{Effect of IPost and BDM on Sarcolemmal Dystrophin}

Dystrophin was exclusively localized in the sarcolemma before ischemia (Figure 4). As demonstrated previously, ${ }^{22,23}$ dystrophin was translocated to the intracellular space during ischemia and was only partially restored to the sarcolemma during control reperfusion. IPost had no significant effect on the recovery of sarcolemmal dystrophin. Treatment with BDM during control reperfusion or IPost significantly increased sarcolemmal dystrophin. Although LY294002 had no significant effect on the restoration of sarcolemmal dystrophin during control reperfusion and reperfusion with BDM, it significantly inhibited the restoration of sarcolemmal dystrophin by IPost and IPost combined with BDM.

\section{Effect of IPost and BDM on Cardiomyocyte Oncosis}

$\mathrm{EB}$ is a membrane impermeable red-fluorescence dye that accumulates into the cells with disrupted plasma membrane. EB staining enables the detection of oncotic cardiomyocytes shortly after reperfusion, when TTC staining underestimates infarcted areas. Before ischemia, there were no cardiomyocytes that accumulated EB (not shown). However, EB was accumulated in cardiomyocytes during control reperfusion (Figure 5A). EB-positive cardiomyocytes had significantly less sarcolemmal dystrophin (not shown). The number of cardiomyocytes positive for EB was not significantly reduced by IPost. In contrast, BDM or IPost with BDM significantly reduced the number of cardiomyocytes positive for EB. LY294002 had no significant effect on the accumulation of EB in cardiomyocytes during control reperfusion and reperfusion with BDM, but significantly increased the number of cardiomyocytes positive for $\mathrm{EB}$ in the hearts undergoing IPost and IPost combined with BDM.

In contrast to the decrease of EB uptake during reperfusion with BDM, the number of cardiomyocytes positive for $\mathrm{EB}$ was not different between the control and BDM groups at the end of reperfusion (Figure 5B). On the contrary, the number of EB-positive cardiomyocytes was significantly decreased in the IPost group at the end of reperfusion. The number of EB-positive cardiomyocytes remained significantly less in the IPost with BDM group compared to the IPost group at the end of reperfusion. Treatment with LY294002 during control reperfusion or reperfusion with BDM had no significant effect on the number of EB-positive cardiomyocytes at the end of reperfusion. However, LY294002 significantly increased the number of EB-positive cardiomyocytes in the hearts that received IPost and IPost combined with BDM.

\section{Effect of IPost and BDM on Cardiomyocyte Apoptosis}

The effect of IPost on cardiomyocyte apoptosis was evaluated by the caspase- 3 activity at the end of IPost, and by use of the TUNEL assay at the end of reperfusion. The caspase-3 activity in the heart was markedly increased during control reperfusion (Figure 6A). IPost significantly decreased caspase-3 activity in the heart. Treatment with BDM during control reperfusion had no significant effect on the activation of caspase-3. However, IPost combined with BDM decreased caspase-3 activity to a level comparable to IPost alone. Treatment with LY294002 had no significant effect on the activation of caspase-3 during control reperfusion and reperfusion with BDM, but it abolished the reduction of the caspase- 3 activity induced by IPost and IPost combined with BDM.

The number of TUNEL-positive cardiomyocytes was significantly decreased by IPost (Figure 6B). While BDM alone had no significant effect on the number of TUNEL-positive cardiomyocytes, IPost combined with BDM decreased TUNEL-positive cardiomyocytes to a level comparable to 

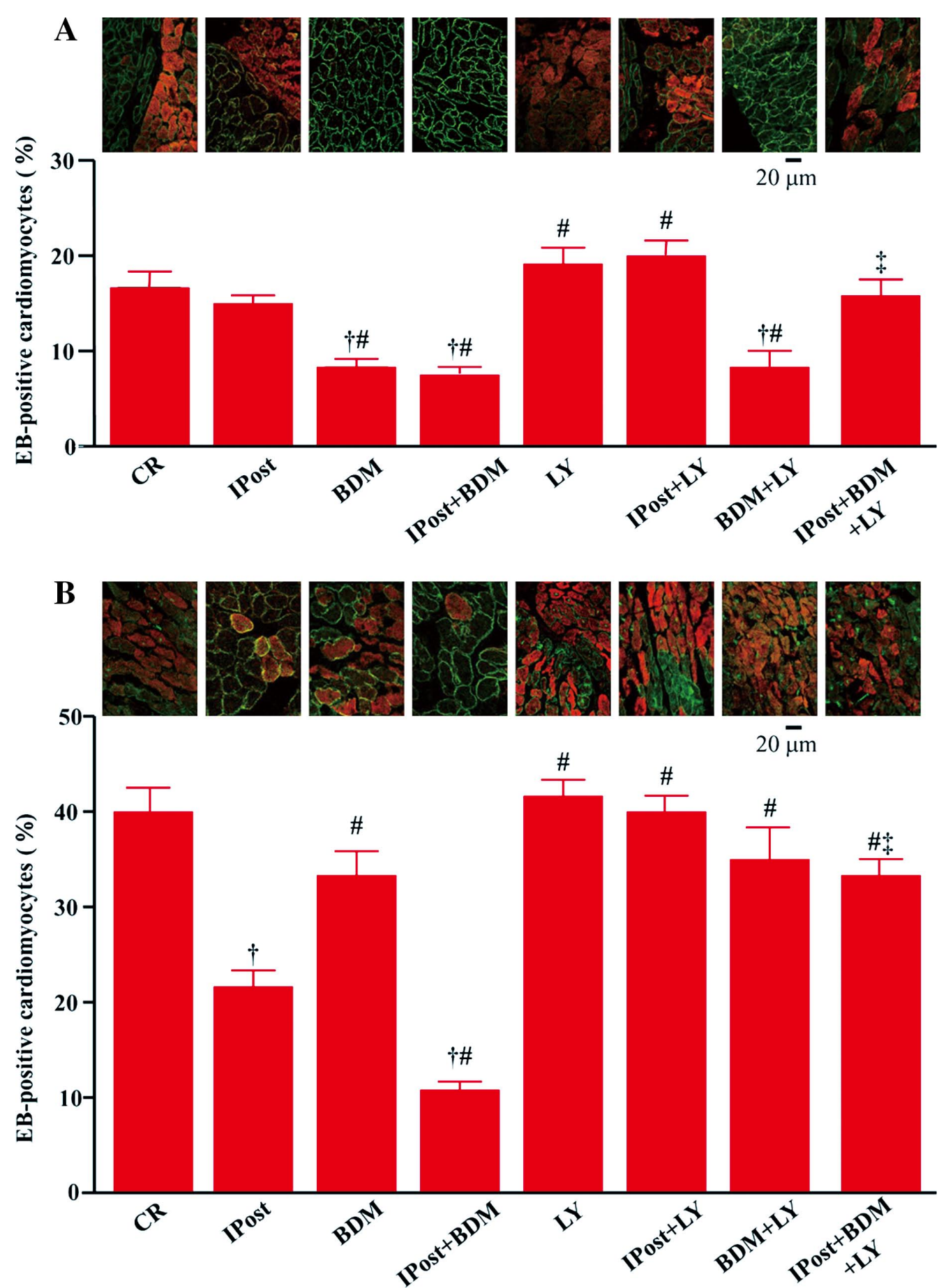

Figure 5. Immunofluorescence images of dystrophin (green) and Evans blue (red). The number of cardiomyocytes positive for Evans blue was quantified at 400 s after control reperfusion (CR), at the end of ischemic postconditioning (IPost), 400 s after reperfusion with 2,3-butanedione monoxime (BDM), at the end of IPost+BDM, 400 s after reperfusion with LY294002 (LY), at the end of IPost $+L Y$, at the end of BDM + LY or at the end of IPost $+B D M+L Y(A)$. Evans blue uptake was also examined $2 \mathrm{~h}$ after reperfu-

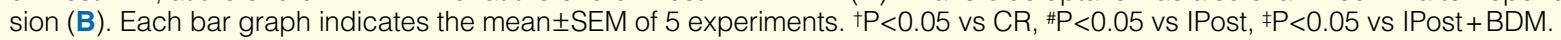

IPost alone. Treatment with LY294002 during control reperfusion or reperfusion with BDM had no significant effect on the number of TUNEL-positive cardiomyocytes, but it abolished the reduction in TUNEL-positive cardiomyocytes observed when the animals were treated with IPost or IPost with BDM.

\section{Effect of IPost and BDM on Infarct Size}

The infarct size was significantly reduced by IPost and it tended to decrease with the use of BDM (Figure 7). When 

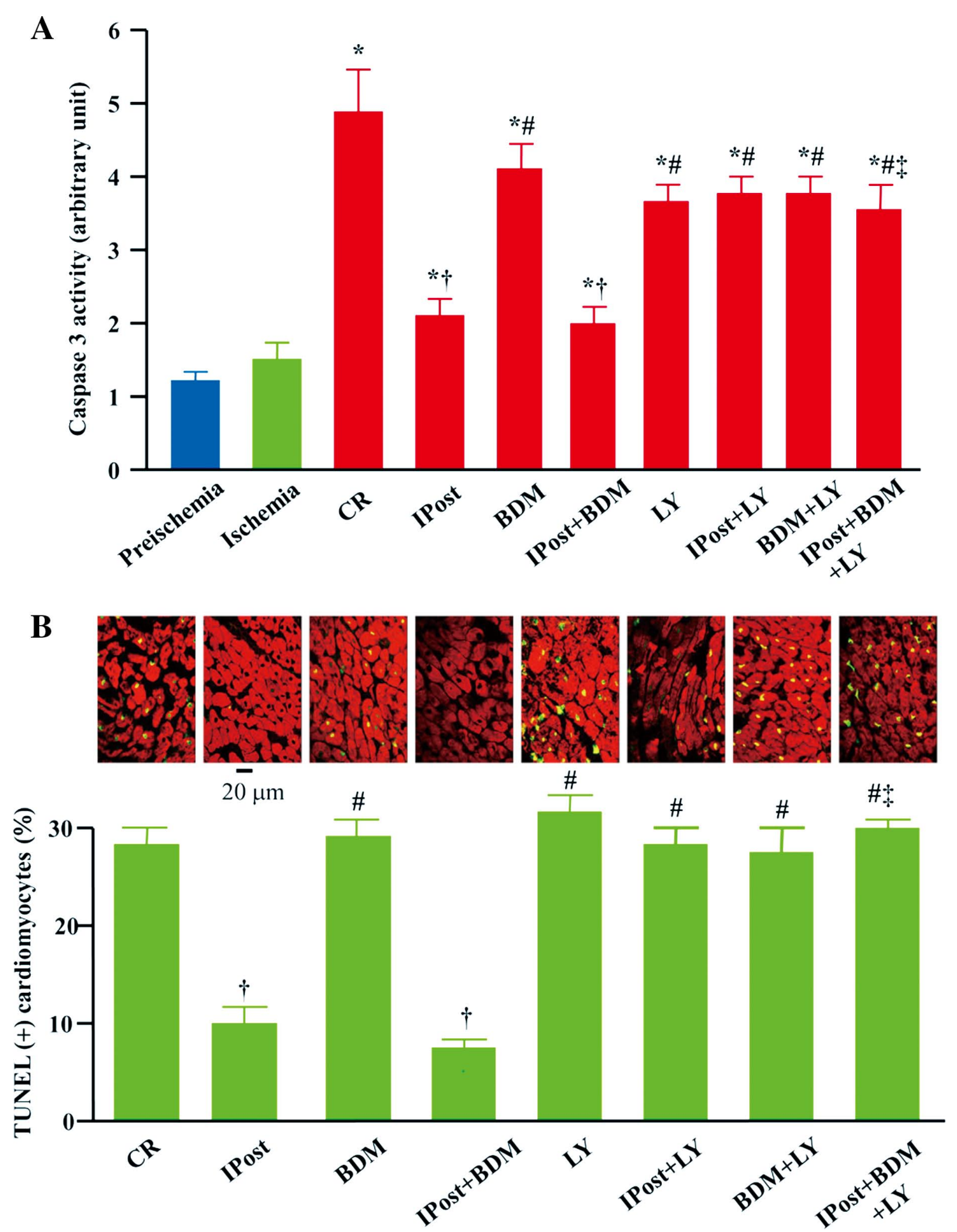

Figure 6. (A) Caspase-3 activity was measured at pre-ischemia, $30 \mathrm{~min}$ after ischemia, $400 \mathrm{~s}$ after control reperfusion (CR), at the end of ischemic postconditioning (IPost), 400 s after reperfusion with 2,3-butanedione monoxime (BDM), at the end of IPost+BDM, 400s after reperfusion with LY294002 (LY), at the end of IPost+LY, at the end of BDM+LY or at the end of IPost $+B D M+L Y$. Each bar graph indicates the mean \pm SEM of 5 experiments. ${ }^{*} P<0.05$ vs pre-ischemia, ${ }^{t} P<0.05$ vs $C R$, ${ }^{\#} P<0.05$ vs IPost, $\neq \mathrm{P}<0.05$ vs IPost +BDM. (B) The number of TUNEL-positive cardiomyocytes was quantified at the end of reperfusion. Each bar graph indicates the mean \pm SEM of 5 experiments. ${ }^{\dagger} \mathrm{P}<0.05$ vs $C R,{ }^{\#} P<0.05$ vs IPost, ${ }^{\mathrm{P}}<0.05$ vs IPost + BDM.

combined, IPost and BDM significantly reduced the infarct size in comparison to IPost alone. Although treatment with LY294002 during control reperfusion or reperfusion with BDM had no significant effect on infarct size, it significantly inhibited the infarct size-limiting effect of IPost and IPost combined with BDM.

\section{Effect of IPost and BDM on LV Function}

There were no significant differences regarding the baseline $\mathrm{LV}$ function in the different groups prior to ischemia (Table). Treatment with BDM abolished the LVDP during control reperfusion or IPost (not shown). Because the LV function demonstrated a maximal recovery at $30 \mathrm{~min}$ after reperfusion 


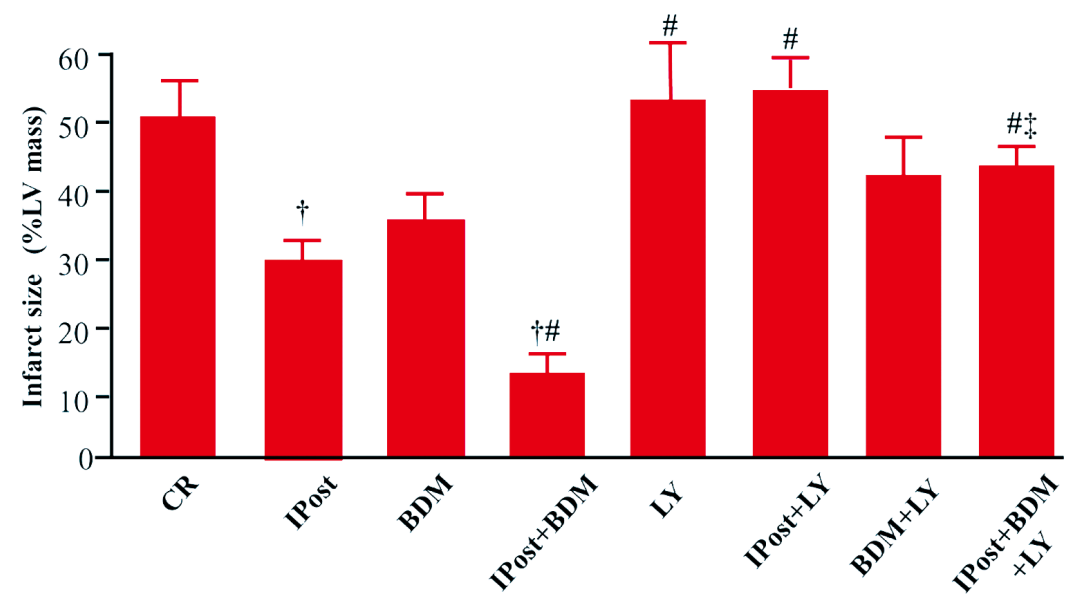

Figure 7. Infarct size was measured at the end of reperfusion in the control reperfusion (CR), ischemic postconditioning (IPost), 2,3-butanedione monoxime (BDM), IPost+BDM, LY294002 (LY), IPost + LY, BDM + LY and IPost + BDM + LY groups. Each bar graph indicates the mean \pm SEM of at least 5 experiments. ${ }^{\mathrm{T}} \mathrm{P}<0.05$ vs $\mathrm{CR},{ }^{\#} \mathrm{P}<0.05$ vs IPost, $\neq \mathrm{P}<0.05$ vs IPost + BDM.

\begin{tabular}{|c|c|c|}
\hline & Baseline & $30 \mathrm{~min} R$ \\
\hline \multicolumn{3}{|l|}{ HR (beats/min) } \\
\hline Control $(n=6)$ & $304 \pm 16$ & $196 \pm 23$ \\
\hline IPost $(n=6)$ & $315 \pm 17$ & $236 \pm 20$ \\
\hline $\operatorname{BDM}(n=5)$ & $335 \pm 17$ & $237 \pm 26$ \\
\hline IPost+BDM $(n=5)$ & $302 \pm 19$ & $280 \pm 12^{*}$ \\
\hline $\operatorname{LY}(n=5)$ & $331 \pm 15$ & $201 \pm 20$ \\
\hline IPost +LY (n=5) & $314 \pm 17$ & $187 \pm 34$ \\
\hline$B D M+L Y(n=5)$ & $320 \pm 18$ & $224 \pm 21$ \\
\hline IPost +BDM +LY $(n=5)$ & $326 \pm 16$ & $193 \pm 20^{\dagger}$ \\
\hline \multicolumn{3}{|l|}{ LVDP (mmHg) } \\
\hline Control $(n=6)$ & $93 \pm 2$ & $34 \pm 7$ \\
\hline IPost $(n=6)$ & $104 \pm 8$ & $70 \pm 8^{*}$ \\
\hline $\operatorname{BDM}(n=5)$ & $99 \pm 6$ & $63 \pm 14$ \\
\hline IPost+BDM $(n=5)$ & $105 \pm 6$ & $100 \pm 9^{*, \star \star}$ \\
\hline $\operatorname{LY}(n=5)$ & $101 \pm 5$ & $35 \pm 3$ \\
\hline IPost +LY (n=5) & $99 \pm 3$ & $38 \pm 4^{\star *}$ \\
\hline$B D M+L Y(n=5)$ & $106 \pm 4$ & $54 \pm 10$ \\
\hline IPost+BDM +LY (n=5) & $94 \pm 3$ & $49 \pm 11^{\dagger}$ \\
\hline \multicolumn{3}{|l|}{ LDEVP $(\mathbf{m m H g})$} \\
\hline Control $(n=6)$ & $8.8 \pm 0.7$ & $68.5 \pm 7.1$ \\
\hline IPost $(n=6)$ & $8.3 \pm 0.9$ & $32.7 \pm 4.5^{\star}$ \\
\hline $\operatorname{BDM}(n=5)$ & $9.6 \pm 0.2$ & $40.6 \pm 7.8^{\star}$ \\
\hline IPost+BDM $(n=5)$ & $8.8 \pm 0.4$ & $18.0 \pm 3.8^{*, * *}$ \\
\hline $\operatorname{LY}(n=5)$ & $8.8 \pm 0.5$ & $62.4 \pm 5.2$ \\
\hline IPost +LY (n=5) & $8.4 \pm 0.2$ & $58.8 \pm 4.1^{\star *}$ \\
\hline$B D M+L Y(n=5)$ & $8.3 \pm 0.6$ & $55.2 \pm 6.1$ \\
\hline IPost +BDM + LY $(n=5)$ & $8.8 \pm 0.7$ & $51.8 \pm 6.1^{* *, \dagger}$ \\
\hline
\end{tabular}

IPost, ischemic postconditioning; BDM, 2,3 butanedione monoxim; $R$, reperfusion; HR, heart rate; LY, LY294002; LVDP, left ventricular developed pressure; LVEDP, left ventricular end-diastolic pressure. Data are expressed as mean \pm SEM.

${ }^{*} \mathrm{P}<0.05$ compared to control, ${ }^{* *} \mathrm{P}<0.05$ compared to IPost, ${ }^{\mathrm{P}} \mathrm{P}<0.05$ compared to IPost+BDM.

in all groups, we compared LV function at this time-point. Only IPost with BDM significantly increased the heart rate compared to control reperfusion. The LVDP was significantly higher, and the LVEDP was significantly lower in the hearts treated with IPost compared to control reperfusion, and these parameters of LV function were further improved by IPost in combination with $\mathrm{BDM}(\mathrm{P}<0.05$ compared to IPost alone). Although treatment with LY294002 during control reperfusion or reperfusion with BDM had no significant effect on heart rate, LVDP or LVEDP, it abrogated the improvement of LV function induced by IPost and IPost combined with BDM.

\section{Discussion}

The salient findings of the present study were as follows: (1) although IPost induced by 20 cycles of 10 -s reperfusion and 10 -s ischemia reduced infarct size and improved LV function compared to control reperfusion, it had no effect on either myocardial ATP, the restoration of sarcolemmal dystrophin or cardiomyocyte oncosis during IPost; (2) the administration of BDM during IPost increased myocardial ATP and facilitated the restoration of sarcolemmal dystrophin, which was associated with a reduction of cardiomyocyte oncosis and infarct size and improvement of LV function; (3) the administration of LY294002 inhibited the phosphorylation of Akt, recovery of ATP and restoration of sarcolemmal dystrophin, and abolished the anti-oncotic effect of IPost combined with BDM. These results suggest that inhibition of contractile activity during IPost prevents cardiomyocyte oncosis, and enhances the infarct size-limiting effect of IPost. Moreover, these effects apparently occur through the PI3Kdependent restoration of sarcolemmal dystrophin.

We used 20 cycles of 10-s reperfusion and 10-s ischemia as an algorithm of IPost in our experimental model. This algorithm was found to be the optimal frequency and duration for reducing infarct size in this experimental model (A.M. and H.O., unpublished data). It is well-established that the algorithm is a critical determinant of the success of the IPost, and that different species and experimental models require different algorithms of IPost to induce optimal cardioprotection. ${ }^{30}$ There is some controversy about whether or not the rat heart can be effectively postconditioned. For example, the rat heart seems to be less sensitive to the cardioprotective effects of IPost. Postconditioning has been reported to not protect isolated and perfused rat hearts, ${ }^{31}$ while also not reducing the infarct size in rat hearts with regional ischemia induced by coronary artery occlusion in vivo. ${ }^{32}$ Although the underlying cause(s) of the disparity in the efficacy of IPost between experiments using the rats is 
unclear, the findings of our study are consistent with the notion that the rat heart can be postconditioned when an appropriate algorithm is used. ${ }^{30}$

The present study suggests that when the heart is undergoing IPost, it is susceptible to oncosis in the presence of contractile activity. We have previously demonstrated that restoration of sarcolemmal dystrophin is crucial for preventing oncosis and reperfusion injury in the ischemic preconditioned rat heart, and that the cardioprotective effect of ischemic preconditioning is dependent on mitochondrial function and ATP generation. ${ }^{24}$ The fact that IPost did not increase myocardial ATP nor facilitate the restoration of sarcolemmal dystrophin suggests that aerobic ATP generation is impaired during IPost. The poor recovery of the ATP level during IPost might also be attributed to energy consumption by contractile activity. Indeed, we observed that the inhibition of the contractile activity by treatment with BDM during IPost increased myocardial ATP and prevented cardiomyocyte oncosis.

The cardioprotective effect of BDM during IPost is thought to be mediated primarily by the inhibition of oncosis. Treatment with BDM during IPost did not increase PI3K activity, as assessed by phosphorylation of Akt compared to IPost alone. Furthermore, the anti-apoptotic effect of IPost with BDM, as evaluated by caspase-3 activity and the TUNEL assay, was not significantly different from IPost alone. These observations suggest that treatment with BDM during IPost did not enhance the anti-apoptotic activity beyond that attributable to IPost alone. BDM is known to inhibit myosin ATPase, thereby diminishing the contractile activity and ATP utilization. ${ }^{33}$ However, BDM exerts multiple side effects such as blocking gap junctional communication ${ }^{34}$ and inhibiting protein phosphatases and adenine nucleotide exchange..$^{35}$ These effects of BDM are thought to preserve ATP and inhibit oncosis during reperfusion, although the anti-oncotic effect of BDM is limited to the period of contractile arrest. In contrast, IPost does not prevent cardiomyocyte oncosis during the procedure, but does prevent the occurrence of oncosis thereafter, presumably by protecting the mitochondria. Thus, administration of BDM during IPost additively or synergistically potentiates the infarct size-limiting effect of IPost by protecting cardiomyocytes depleted of ATP and dystrophin from prematurely dying from oncosis.

The present study suggests that PI3K plays a crucial role in IPost-induced anti-apoptotic and anti-oncotic effects, because when LY294002 was used to inhibit PI3K activity, the recovery of ATP and restoration of sarcolemmal dystrophin were decreased and the anti-oncotic and the anti-apoptotic effects of IPost were blocked. These observations are consistent with previous studies ${ }^{28,29}$ demonstrating that the cardioprotective effect of IPost is mediated by PI3K in the isolated and perfused rat heart. In contrast, although treatment with LY294002 during control reperfusion or reperfusion with BDM inhibited PI3K activity, it did not significantly decrease myocardial ATP, nor did it increase the caspase-3 activity, TUNEL-positive cardiomyocytes, EB uptake by cardiomyocytes, or infarct size compared to the heart with control reperfusion or reperfusion with BDM. This finding suggests that there might be other signaling pathways than PI3K-Akt to protect the mitochondria in the presence of LY294002 in the control or BDM-treated heart. In contrast, IPost-induced cardioprotection is dependent on PI3K and can not be compensated for by other pathways.

The present study indicates that the inhibition of the contractile activity and preserving ATP during IPost might rep- resent a promising approach for potentiating cardioprotection against reperfusion injury in patients with MI. It has been demonstrated that IPost can be used to effectively reduce myocardial injury and improve the prognosis for patients receiving percutaneous coronary interventions for acute MI. 2,36 However, there have so far been no systematic investigations as to the effect of contractile blockers on the cardioprotection induced by IPost. Although BDM is the most commonly used contractile blocker in animal studies, the clinical use of BDM might not be feasible. A potential alternative to BDM is the use of $\beta$-blockers or calcium channel blockers such as verapamil, which inhibit contractile activity and preserve ATP similar to BDM. Future studies using these pharmacological tools are warranted to verify the hypothesis that inhibiting contractility and preserving ATP is a universal mechanism for enhancement of the cardioprotective effect of IPost.

We should be cautious to use the word "oncosis". Our EB dye uptake method does not differentiate oncosis from necrosis, which is defined by cell death with disrupted plasma membrane by any causes. It is unlikely that cardiomyocytes died from apoptosis and undergo a secondary necrotic change with sarcolemmal disruption within $400 \mathrm{~s}$ after reperfusion. Therefore, the majority, if not all, cardiomyocytes accumulated EB should be killed by oncosis at this early stage after reperfusion. However, we are uncertain whether cardiomyocytes positive for EB were killed solely by oncosis $2 \mathrm{~h}$ after reperfusion. Therefore, it might be more appropriate to use the word "necrosis" when cardiomyocyte death is evaluated by EB dye uptake or TTC staining $2 \mathrm{~h}$ after reperfusion.

In conclusion, the inhibition of the contractile activity during IPost prevents cardiomyocyte oncosis and enhances the infarct size-limiting effect of IPost through PI3K-dependent restoration of sarcolemmal dystrophin. Although it remains to be investigated whether the inhibition of the contractile activity during IPost enhances the cardioprotection afforded by IPost in humans, temporarily maintaining contractile arrest in the previously ischemic myocardium during IPost might therefore represent a novel strategy to enhance the cardioprotective effect of IPost.

\section{Acknowledgement}

This study was supported, in part, by a Research Grant (No.19590838) from the Ministry of Education, Science, and Culture of Japan.

\section{References}

1. Zhao ZQ, Vinten-Johansen J. Postconditioning: Reduction of reperfusion-induced injury. Cardiovasc Res 2006; 70: 200-211.

2. Vinten-Johansen J, Yellon DM, Opie LH. Postconditioning: A simple, clinically applicable procedure to improve revascularization in acute myocardial infarction. Circulation 2005; 112: 2085-2088.

3. Downey JM, Cohen MV. Why do we still not have cardioprotective drugs? Circ J 2009; 73: 1171-1177.

4. Zhao ZQ, Corvera JS, Halkos ME, Kerendi F, Wang NP, Guyton $\mathrm{RA}$, et al. Inhibition of myocardial injury by ischemic postconditioning during reperfusion: Comparison with ischemic preconditioning. Am J Physiol Heart Circ Physiol 2003; 285: H579-H588.

5. Kin H, Zhao ZQ, Sun HY, Wang NP, Corvera JS, Halkos ME, et al. Postconditioning attenuates myocardial ischemia-reperfusion injury by inhibiting events in the early minutes of reperfusion. Cardiovasc Res 2004; 62: 74-85.

6. Fujita M, Asanuma H, Hirata A, Wakeno M, Takahama H, Sasaki $\mathrm{H}$, et al. Prolonged transient acidosis during early reperfusion contributes to the cardioprotective effects of postconditioning. Am J Physiol Heart Circ Physiol 2007; 292: H2004-H2008.

7. Crompton M, Virji S, Doyle V, Johnson N, Ward JM. The mitochondrial permeability transition pore. Biochem Soc Symp 1999; 66: $167-179$.

8. Hirsch T, Susin SA, Marzo I, Marchetti P, Zamzami N, Kroemer G. Mitochondrial permeability transition in apoptosis and necrosis. 
Cell Biol Toxicol 1998; 14: $141-145$.

9. Halestrap AP, Clarke SJ, Javadov SA. Mitochondrial permeability transition pore opening during myocardial reperfusion--a target for cardioprotection. Cardiovasc Res 2004; 61: 372-385.

10. Miura T, Miki T. GSK-3 $\beta$, a therapeutic target for cardiomyocyte protection. Circ J 2009; 73: $1184-1192$.

11. Davidson SM, Hausenloy D, Duchen MR, Yellon DM. Signalling via the reperfusion injury signalling kinase (RISK) pathway links closure of the mitochondrial permeability transition pore to cardioprotection. Int J Biochem Cell Biol 2006; 38: 414-419.

12. Hausenloy DJ, Tsang A, Yellon DM. The reperfusion injury salvage kinase pathway: A common target for both ischemic preconditioning and postconditioning. Trends Cardiovasc Med 2005; 15: 6975 .

13. Majno G, Joris I. Apoptosis, oncosis, and necrosis: An overview of cell death. Am J Pathol 1995; 146: 3-15.

14. Garcia-Dorado D, Theroux P, Duran JM, Solares J, Alonso J, Sanz E, et al. Selective inhibition of the contractile apparatus: A new approach to modification of infarct size, infarct composition, and infarct geometry during coronary artery occlusion and reperfusion. Circulation 1992; 85: 1160-1174.

15. Schlack W, Uebing A, Schafer M, Bier F, Schafer S, Piper HM, et al. Regional contractile blockade at the onset of reperfusion reduces infarct size in the dog heart. Pflugers Arch 1994; 428: 134-141.

16. Ganote C, Armstrong S. Ischaemia and the myocyte cytoskeleton Review and speculation. Cardiovasc Res 1993; 27: 1387-1403.

17. Armstrong SC, Latham CA, Shivell CL, Ganote CE. Ischemic loss of sarcolemmal dystrophin and spectrin: Correlation with myocardial injury. J Mol Cell Cardiol 2001; 33: 1165-1179.

18. Otani H. Ischemic preconditioning: From molecular mechanisms to therapeutic opportunities. Antioxid Redox Signal 2008; 10: 207 247.

19. Rybakova IN, Patel JR, Ervasti JM. The dystrophin complex forms a mechanically strong link between the sarcolemma and costameric actin. J Cell Biol 2000; 150: 1209-1214.

20. Petrof BJ, Shrager JB, Stedman HH, Kelly AM, Sweeney HL. Dystrophin protects the sarcolemma from stresses developed during muscle contraction. Proc Natl Acad Sci USA 1993; 90: 3710-3714.

21. Straub V, Bittner RE, Leger JJ, Voit T. Direct visualization of the dystrophin network on skeletal muscle fiber membrane. J Cell Biol 1992; 119: 1183-1191.

22. Kyoi S, Otani H, Sumida T, Okada T, Osako M, Imamura H, et al Loss of intracellular dystrophin: A potential mechanism for myocardial reperfusion injury. Circ J 2003; 67: 725-727.

23. Kido M, Otani H, Kyoi S, Sumida T, Fujiwara H, Okada T, et al. Ischemic preconditioning-mediated restoration of membrane dystrophin during reperfusion correlates with protection against contraction-induced myocardial injury. Am J Physiol Heart Circ Physiol 2004; 287: H81-H90.

24. Kyoi S, Otani H, Hamano A, Matsuhisa S, Akita Y, Fujiwara H, et al. Dystrophin is a possible end-target of ischemic preconditioning against cardiomyocyte oncosis during the early phase of reperfusion. Cardiovasc Res 2006; 70: 354-363.

25. Malik G, Gorbounov N, Das S, Gurusamy N, Otani H, Maulik N, et al. Ischemic preconditioning triggers nuclear translocation of thioredoxin and its interaction with Ref-1 potentiating a survival signal through the PI-3-kinase-Akt pathway. Antioxid Redox Signal 2006; 8: $2101-2109$.

26. Uchiyama T, Otani H, Okada T, Ninomiya H, Kido M, Imamura H, et al. Nitric oxide induces caspase-dependent apoptosis and necrosis in neonatal rat cardiomyocytes. J Mol Cell Cardiol 2002; 34: $1049-1061$.

27. Otani H, Matsuhisa S, Akita Y, Kyoi S, Enoki C, Tatsumi K, et al. Role of mechanical stress in the form of cardiomyocyte death during the early phase of reperfusion. Circ J 2006; 70: 1344-1355.

28. Tsang A, Hausenloy DJ, Mocanu MM, Yellon DM. Postconditioning: A form of "modified reperfusion" protects the myocardium by activating the phosphatidylinositol 3-kinase-Akt pathway. Circ Res 2004; 95: 230-232.

29. Bopassa JC, Ferrera R, Gateau-Roesch O, Couture-Lepetit E, Ovize M. PI 3-kinase regulates the mitochondrial transition pore in controlled reperfusion and postconditioning. Cardiovasc Res 2006; 69: $178-185$.

30. van Vuuren D, Lochner A. Ischaemic postconditioning: From bench to bedside. Cardiovasc J Afr 2008; 19: 311-320.

31. Kaljusto ML, Mori T, Mohammad Husain Rizvi S, Galagudza M, Frantzen ML, Valen G, et al. Postconditioning in rats and mice. Scand Cardiovasc J 2006; 40: 334-341.

32. Dow J, Kloner RA. Postconditioning does not reduce myocardial infarct size in an in vivo regional ischemia rodent model. J Cardiovasc Pharmacol Ther 2007; 12: 153-163.

33. Higuchi H, Takemori S. Butanedione monoxime suppresses contraction and ATPase activity of rabbit skeletal muscle. J Biochem 1989; 105: 638-643.

34. Verrecchia F, Herve JC. Reversible blockade of gap junctional communication by 2,3-butanedione monoxime in rat cardiac myocytes. Am J Physiol 1997; 272: C875-C885.

35. Stapleton MT, Fuchsbauer CM, Allshire AP. BDM drives protein dephosphorylation and inhibits adenine nucleotide exchange in cardiomyocytes. Am J Physiol 1998; 275: H1260-H1266.

36. Staat P, Rioufol G, Piot C, Cottin Y, Cung TT, L'Huillier I, et al. Postconditioning the human heart. Circulation 2005; 112: $2143-$ 2148 . 